\title{
El proceso de creación del curso de especialización en Enfermería Obstétrica, modalidad de residencia, en la red municipal de salud de Rio de Janeiro, Brasil
}

\author{
The creation process of the specialization course in Obstetric Nursing, \\ residency modality, in the municipal health network of Rio de Janeiro, Brazil
}

\section{O processo de criação do curso de especialização em Enfermagem Obstétrica, modalidade de residência, na rede municipal de saúde do Rio de Janeiro, Brasil}

\section{Adriana Lenho de Figueiredo Pereira1, Ricardo José Oliveira Mouta², Dayane Silva Palha Barbosa ${ }^{3}$}

\footnotetext{
${ }^{1}$ Doutora em Enfermagem. Professora Adjunta do Departamento de Enfermagem Materno-Infantil da Faculdade de Enfermagem da Universidade do Estado do Rio de Janeiro. Rio de Janeiro, Rio de Janeiro, Brasil.Correo electrónico: adrianalenho.uerj@gmail.com

${ }^{2}$ Doutor em Enfermagem. Professor Adjunto do Departamento de Enfermagem Materno-Infantil da Faculdade de Enfermagem da Universidade do Estado do Rio de Janeiro. Rio de Janeiro, Rio de Janeiro, Brasil. Correo electrónico: ricardomouta@hotmail.com

${ }^{3}$ Acadêmica de Enfermagem. Bolsista de Iniciação Científica da Universidade do Estado do Rio de Janeiro. Rio de Janeiro, Rio de Janeiro, Brasil. Correo electrónico: dayanespb@gmail.com
}

Cómo citar este artículo en edición digital: Pereira, A.L.F., Mouta, R.J.O., \& Barbosa, D.S.P.(2018). El proceso de creación del curso de especialización en Enfermería Obstétrica, modalidad de residencia, en la red municipal de salud de Rio de Janeiro, Brasil. Cultura de los Cuidados (Edición digital), 22(51). Recuperado de <http://dx.doi.org/10.14198/cuid.2018.51.13> Correspondencia: Faculdade da Enfermagem-Universidad do Estado de Rio de JaneiroUERJ/Brasil.Boulevard 28 setembre 157, sala 700-Vila Isabel.Rio de Janeiro-RJ-Brasil-CEP:20551-030 Correo electrónico: adrianalenho.uerj@gmail.com Recibido: 20/10/2017; Aceptado: 11/02/2018.

\begin{abstract}
The aim of this study is to describe the creation process of the specialization course in Obstetric Nursing, residence modality, in the municipal health network of Rio de Janeiro. This is a qualitative, exploratory, descriptive study, of social historical nature. The analysis corpus of this research were primary sources that consisted of written documents and oral testimony that
\end{abstract}

have been produced using semi-structured interviews with the professional group SMS-RJ and teachers of ENF/UERJ, who worked directly in the design and implementation the proposal of the course from 2003 to 2005. The results showed that the health policy in the country looked for the qualification of health care of women, reducing maternal and neonatal mortality and changing the care model toward the humanization of childbirth and birth. Due to shortage of obstetric nurses in the municipal 
health network, there was a partnership of SMSRJ and ENF / UERJ for the formation of these professionals as an opportunity for expansion of their activities. We conclude that the process of creation of this specialization model in the municipal health of Rio de Janeiro had challenges for managers and teachers for its implementation.

Keywords: Nursing, Obstetric Nursing, History of Nursing, Nursing Education

\section{RESUMEN}

El objetivo de este estudio es describir el proceso de creación del curso de especialización en Enfermería Obstetricia, modalidad de residencia, en la red municipal de salud municipal de Río de Janeiro. Se trata de una investigación de carácter cualitativo, exploratorio, descriptivo, histórico social. El corpus de análisis de esta investigación fueron fuentes primarias que consistieron en documentos escritos y testimonios orales que se han producido a través de entrevistas semiestructuradas con el grupo profesional de SMS-RJ y los maestros de la ENF / UERJ, que actuaron directamente en la concepción y ejecución de la propuesta del curso de 2003 a 2005. Los resultados mostraron que la política de salud en el país buscaba la calificación del cuidado de la salud de la mujer, la reducción de la mortalidad materna y neonatal y el cambio del modelo asistencial en dirección a la humanización del parto y nacimiento. Debido a la escasez de enfermeras obstétricas en la red municipal de salud, hubo una parcería de SMSRJ y ENF / UERJ para la formación de estas profesionales como una oportunidad para la expansión de sus actividades. Llegamos a la conclusión de que el proceso de creación de este modelo de especialización en la red municipal de Río de Janeiro presentó desafíos para los directores y maestros durante su implementación.

\section{INTRODUÇÃO}

No Brasil, o ensino de Enfermagem Obstétrica apresentou mudanças em sua configuração que foram decorrentes do processo de transformações histórico-sociais da profissão e
Palabras Clave: Enfermería, Enfermería Obstétrica, Historia de la Enfermería, Educación en Enfermería

\section{RESUMO}

O objetivo deste estudo é descrever o processo de criação do curso de especialização em Enfermagem Obstétrica, modalidade de residência, na rede municipal de saúde do Rio de Janeiro. Trata-se de um estudo qualitativo, exploratório, descritivo, de natureza histórica social. O corpus de análise desta pesquisa foram fontes primárias que se constituíram de documentos escritos e depoimentos orais que foram produzidos por meio de entrevistas semiestruturadas realizadas com o grupo de profissionais da SMS-RJ e docentes da ENF/UERJ que atuaram diretamente na concepção e execução da proposta do curso, no período de 2003 a 2005. Os resultados indicam que a política de saúde no país buscava a qualificação da assistência à saúde da mulher, a redução da mortalidade materna e neonatal e a mudança do modelo assistencial em direção à humanização do parto e nascimento. Devido à carência de enfermeiras obstétricas na rede municipal de saúde, houve a parceria da SMS-RJ e da ENF/UERJ para a formação dessas profissionais como uma oportunidade de ampliação da atuação delas. Conclui-se que o processo de criação deste modelo de especialização na rede municipal do Rio de janeiro apresentou desafios para os gestores e docentes durante sua implementação.

Palavras Chave: Enfermagem, Enfermagem Obstétrica, História da Enfermagem, Educação em Enfermagem

do sistema educacional e de saúde (Erdmann, Fernandes \& Teixeira, 2011). Após a Reforma Universitária Brasileira, ocorrida no ano 1968, houve a instituição de um novo currículo mínimo para a graduação em Enfermagem no ano de 1972, consequente ao Parecer do 
Conselho Federal de Educação (CFE) n n 163/72 e da Resolução do CFE $n^{\circ} 04 / 72$, que conformou uma nova estrutura curricular da graduação e a segmentou em dois momentos: o ciclo préprofissional, constituído basicamente por conteúdos das ciências básicas e sociais, e o ciclo profissional, organizado em disciplinas específicas da Enfermagem e complementado por um seguimento optativo, representado por habilitações específicas, como a Enfermagem de Saúde Pública, a Enfermagem Obstétrica e a Enfermagem Médico-Cirúrgica (Pereira, 2013).

Esta conformação curricular atendia à organização do sistema de saúde da década de 1970, cujas ações de saúde eram verticalizadas e predominantemente curativas, com relevância nos atendimentos ambulatoriais e hospitalares. Para as mulheres, a ênfase era no atendimento ao período reprodutivo feminino, sendo criados o Programa de Saúde Materno-Infantil, em 1975, e o Programa de Prevenção à Gravidez de Alto Risco, em 1978. Na vigência destes programas, havia uma perspectiva assistencial pautada no excesso de intervenções sobre o corpo feminino, o que ocasionou a elevação progressiva da cesariana e da esterilização como método contraceptivo preferencial (Souza \& Tyrrell, 2011). Na vigência da Reforma Sanitária, nos anos de 1980, houve a participação dos movimentos sociais feministas que intentavam a reformulação programática por meio da valorização da mulher como sujeito nas práticas de saúde e a inclusão das ações voltadas para os problemas clínico-ginecológicos, reprodutivos e de controle da fertilidade, entre outras. Estas proposições balizaram a formulação do Programa de Atenção Integral à Saúde da Mulher (PAISM), considerado um marco na concepção da saúde integral em todo o ciclo vital e com perspectiva na cidadania feminina (Rattner, 2014).

Ainda nos anos de 1980, a saúde da mulher passou a integrar a agenda internacional devido aos altos índices de morbimortalidade materna e perinatal, sobretudo nos países em desenvolvimento. A Organização Mundial de Saúde (OMS) realiza conferências para enfrentar esta problemática, com destaque para a Conferência Internacional Tecnologia Apropriada para o Nascimento, realizada em Fortaleza, capital do estado do Ceará, na região nordeste do Brasil, em 1985. Esta conferência estabeleceu a parturição deve ser concebida como um processo fisiológico, com estímulo à autonomia da mulher e ao direito ao acompanhante, e propôs várias recomendações para uma assistência com menor grau de intervenção. Desde então, estas recomendações influenciam as discussões sobre os prejuízos das excessivas intervenções e tecnologias utilizadas no processo parturitivo, Oliveira et al. (2014).

Em 1994, o ensino de Enfermagem Obstétrica passa por outra modificação com o parecer CFE/MEC n. 314, homologado pelo Ministério da Educação (MEC) por meio da Portaria $n^{\circ}$ 1.721, de 15 de dezembro de 1994, que estabeleceu os mínimos de conteúdo do curso de graduação em Enfermagem, com duração de oito a doze semestres e com foco na formação do enfermeiro generalista. A formação especializada passou a ser realizada apenas por meio dos cursos de pós-graduação lato sensu, também denominados de cursos de especialização (Silva, Fernandes, Teixeira, \& Silva, 2010). A partir de 1998, o Ministério da Saúde (MS) incentiva o parto normal em virtude do aumento progressivo das cesarianas; promove a melhoria dos indicadores de morbimortalidade materna e neonatal e a atuação das enfermeiras obstétricas no Sistema Único de Saúde (SUS). Para tal, houve a formalização de convênios com as Instituições de Ensino Superior de Enfermagem e disponibilizou apoio financeiro e assessoria técnica para a realização de cursos de especialização em Enfermagem Obstétrica, sendo desenvolvidos 76 cursos e qualificadas 1.366 enfermeiras obstétricas em todo país, no período de 1999 a 2004 (Costa \& Schirmer, 2012).

Essa iniciativa governamental foi impulsionada pela criação do Programa de Humanização no Pré-Natal e Nascimento (PHPN), instituído pelas Portarias GM/569, 570, 571 e 572/2000, e pelas metas estabelecidas pelos Objetivos de Desenvolvimento do Milênio das Nações Unidas também no ano 2000, que foram assumidas pelo Brasil (Ministério da Saúde, 2000).

O currículo desses cursos de especialização apresentava um terço da carga horária para os conteúdos teóricos das disciplinas de Obstetrícia Fundamental, Enfermagem Obstétrica e Enfermagem Perinatal, e também dois terços de atividades práticas nos serviços de saúde. Os programas dos cursos estavam baseados na 
abordagem de gênero, sexualidade e saúde reprodutiva, com enfoque nos direitos de cidadania, sejam eles políticos, jurídicos, trabalhistas ou sociais; na discussão dos indicadores epidemiológicos de saúde e avaliação de qualidade da assistência; nos princípios da bioética; na discussão sobre as questões éticas e legais do exercício profissional, além das políticas públicas voltadas para a saúde das mulheres.

Apesar desse incentivo, o ensino da Enfermagem Obstétrica enfrentou barreiras institucionais, sobretudo na assistência ao parto normal. Nos cenários de prática hospitalar ainda prevalece o modelo intervencionista e medicalizado, o que suscita uma contradição entre o paradigma norteador do currículo e o paradigma assistencial dominante, que pode catalisar conflitos profissionais e interferir na qualidade da formação destas profissionais, Lima et al. (2015).

Por outro lado, o quantitativo de enfermeiras obstétricas ainda é reduzido no país e há clara necessidade de uma qualificação de excelência, capaz de conferir a estas especialistas os conhecimentos, competências, habilidades e atitudes necessárias para atuar com segurança e dispor de plenas condições de desenvolver seu trabalho no âmbito da equipe assistencial da área obstétrica (Amorim \& Gualda, 2011). Em virtude da necessidade de ampliar esse quantitativo, a Secretaria Municipal de Saúde do Rio de Janeiro (SMS-RJ) também estabeleceu incentivos para a formação e a atuação dessas profissionais nas maternidades públicas. Dentre outros incentivos, foi constituída parceria com a Faculdade de Enfermagem da Universidade do Estado do Rio de Janeiro (ENF/UERJ) para criar o curso de Especialização em Enfermagem Obstétrica na Modalidade de Residência em 2003. O curso iniciou em março do ano 2004, tendo como cenários de ensino em serviço uma Casa de Parto e quatro maternidades integrantes da rede pública de saúde, e está em funcionamento regular desde então (Pereira \& Nicácio, 2014).

A formação teórico-prática ocorre no período de dois anos, com carga horária de 60 horas semanais, e há disponibilização de dez vagas e bolsas auxílio a cada ano, que são custeadas pela SMS-RJ. A ENF/UERJ realiza a coordenação pedagógica e a certificação das enfermeiras residentes. Esta residência em Enfermagem Obstétrica foi pioneira no Estado do Rio de Janeiro, em relação à sua configuração como curso pós-graduação lato sensu vinculado à unidade acadêmica, o que garante a certificação e o exercício legal de suas egressas na especialidade (Pereira \& Nicácio, 2014).

Os treinamentos de enfermeiras em serviço, assemelhados à residência médica, existem no país desde os anos de 1960, mas não dispunham de legislação regulamentadora no MEC. Apesar desta ausência, houve expansão destes treinamentos, denominados de residência em Enfermagem, vinculados às instituições não educacionais e com fragilidades em seus programas de ensino e supervisão pedagógica (Rosa \& Lopes, 2010). Esta ausência de vinculação às Instituições de Ensino Superior (IES) fez com que a formação das enfermeiras obstétricas ocorresse por meio dos cursos de especialização ofertados pelas IES de Enfermagem.

Em relação à ENF/UERJ, a qualificação de enfermeiras obstétricas ocorre desde anos 1970. Inicialmente, por meio da formação complementar ao Curso de Graduação, denominada de habilitação em Enfermagem Obstétrica. A partir de 1992, essa qualificação passa a ser realizada apenas por meio da especialização em Enfermagem Obstétrica, que é um curso de pós-graduação lato sensu e com carga horária de $420 \mathrm{~h}$.

Em relação à residência em Enfermagem, a regulamentação como modalidade de ensino de pós-graduação lato sensu pelo MEC ocorre a partir da Lei $n^{\circ} 11.129$, de 30 de junho de 2005, quando foi instituída a Residência em Área Multiprofissional e Profissional da Saúde, que é voltada para a educação em serviço de profissionais da área de saúde, com exceção dos profissionais médicos. Esta lei também cria a Comissão Nacional de Residência Multiprofissional em Saúde - CNRMS, vinculada ao MEC. Desde então, os programas residência nas áreas de saúde são desenvolvidos com duração mínima de dois anos e carga horária de 5760 h, com ênfase nas áreas prioritárias para o SUS, que inclui a área da enfermagem obstétrica (Lei $n^{0} 11.129$, de 30 de junho de 2005, 2005). 
Em decorrência da política de reorientação da formação dos trabalhadores em saúde, sobretudo para as áreas estratégicas do SUS, o Ministério da Saúde estabeleceu novo incentivo à formação de enfermeiras obstétricas por meio da criação do Programa Nacional de Residência em Enfermagem Obstétrica (PRONAENF), em parceria com o MEC, no ano de 2012 (Secretaria de Gestão do Trabalho e da Educação na Saúde, 2012). Este PRONAENF objetiva incentivar a formação de especialistas na modalidade residência para atuar no cuidado à saúde da mulher nos processos de saúde reprodutiva, prénatal, parto e nascimento, ao puerpério e à família, orientados pelas políticas de saúde vigentes no sistema de saúde brasileiro (Secretaria de Gestão do Trabalho e da Educação na Saúde, 2012). Assim, os cursos de especialização na modalidade de residência passam a receber incentivo ministerial no país com intuito de aumentar o quantitativo das enfermeiras obstétricas e contribuir com as ações de estímulo do parto normal e melhoria dos indicadores da assistência obstétrica no país.

Nesse sentido, a presente pesquisa tem como objetivo descrever o processo de criação do curso de especialização em Enfermagem Obstétrica, modalidade de residência, na rede municipal de saúde do Rio de Janeiro.

\section{METODOLOGIA}

Trata-se de um estudo qualitativo, exploratório, descritivo, de natureza histórica social. O corpus de análise desta pesquisa foram fontes primárias constituídas por documentos escritos e depoimentos orais.

Foram selecionados dois documentos institucionais, sendo o primeiro representado pelo Processo SMS-RJ 09/0229960/03, título “Ofício S/CRH/DRH/n³71/2003”, assunto "Encaminhamentos do Projeto de Concessão de Bolsas para Pós-Graduação em Enfermagem Obstétrica nos moldes de residência 2004”, de 22 de outubro de 2003, 16 folhas e um anexo, constituído pela cópia do Processo SMS-RJ 09/021616-03, título “Ofício S/CRH/DRH/n²65/2003”, assunto "Enc. Planilha de Custo/2004 para concessão de bolsas, etc...”, de 14 de julho de 2003, nove folhas, acervo da Direção da ENF/UERJ. O segundo documento selecionado foi o Processo UERJ, no 7288/2005, intitulado "Projeto Prop. Criação Curso Esp. Enf. Obstétrica Mod.
Residência”, de 18 de agosto de 2005, 105 folhas, acervo da Sub-reitoria de Pós-graduação e Pesquisa desta universidade (SR-2).

Para a análise desses documentos, foi utilizado um roteiro estruturado e constituído de duas partes: a primeira concernente à especificação do documento e a outra parte que tratou da descrição dos fatos, acontecimentos e contexto da criação do curso na modalidade de residência.

Os depoimentos orais foram produzidos por meio de entrevistas semiestruturadas realizadas com o grupo de profissionais da SMSRJ e de docentes da FENF/UERJ que atuaram diretamente na concepção e execução da proposta do curso, no período de 2003 a 2005. Este grupo foi representado pelo Coordenador de Recursos Humanos e a Assessora da Coordenação do Atendimento Integral à Saúde da SMS-RJ e pelos docentes que exerciam os cargos de direção e vice-direção da unidade acadêmica nesse período, bem como a coordenadora do curso de especialização em Enfermagem Obstétrica (420h) e três docentes do Departamento de Enfermagem MaternoInfantil que também desenvolviam atividades de qualificação de pessoal de Enfermagem, em parceria com a SMS-RJ na época. O período de coleta de dados ocorreu de abril a agosto de 2014.

As fontes documentais e orais, a partir da análise documental e da transcrição das entrevistas, foram consolidadas como um conjunto de dados textuais, o corpus da análise textual, e foram analisados por meio da análise temática, que é estabelecida pelas fases da préanálise, exploração do material e tratamento dos resultados obtidos e interpretação (Minayo, 2006).

Para preservar o anonimato das participantes, conforme indicação do Comitê de Ética em Pesquisa que emitiu o parecer sobre o estudo, os depoimentos receberam a codificação pela ordem de concessão das entrevistas (E1, E2, E3...) e pela função exercida na época da criação do curso, como coordenação da SMS ou docente. O estudo foi aprovado pelo CEP da Secretaria Municipal de Saúde (SMS-RJ), parecer $n^{\circ}$ 70A/2013.

\section{RESULTADOS E DISCUSSÃO}


A criação do curso de residência em Enfermagem Obstétrica: motivações e desafios

Na época da criação do curso, a política de saúde no país buscava a qualificação da assistência à saúde da mulher, a redução da mortalidade materna e neonatal e a mudança do modelo assistencial em direção à humanização do parto e nascimento. Para tal, ocorreram estímulos para a formação e assistência das enfermeiras obstétricas, como os cursos de especialização financiados pelo Ministério da Saúde, entre 1999 e 2004, e a criação dos Centros de Parto Normal em 1999.

Nessa perspectiva, a SMS-RJ já havia estabelecido ações para a melhoria da assistência obstétrica em sua rede assistencial, como o estímulo da inserção de enfermeiras obstétricas nas maternidades públicas e a construção de uma Casa de Parto, o que demandava a ampliação do quantitativo de enfermeiras obstétricas capacitadas. Este contexto político foi mencionado nas justificativas descritas nos dois processos institucionais analisados, bem como nos relatos dos entrevistados:

[...] no intuito de estimular a entrada de novas enfermeiras especializadas para atuarem em Maternidades e Casas de Parto do município, de acordo com as diretrizes do programa de humanização do pré-natal, parto e nascimento, a Superintendência de Saúde Coletiva da SMS/RJ solicitou uma parceria com a ENF/UERJ em 2003, para a criação do Curso de Especialização em Enfermagem Obstétrica na Modalidade de Residência (Processo UERJ, p. 3).

[...] Era exatamente a questão da qualificação dos enfermeiros obstetras visando à mudança de paradigma de atenção à mulher, que não ficasse mais naquela questão da medicalização, mas de uma atenção mais humanística, mais voltada para a mulher, a pessoa como cidadã.... Então, o curso foi concebido num momento muito importante porque ele tinha uma relação político-social. Político, porque havia alguns editais no Ministério da Saúde, na época, específicos para Enfermagem Obstétrica, principalmente, para as universidades públicas federais e a nossa tinha uma bagagem de trabalho importante no Rio de Janeiro, junto e articulada com a SMS. (E1, docente)
A medicalização é compreendida como o processo de controle social pelo qual os eventos naturais do ciclo vital e os desvios no comportamento do corpo são considerados como doenças ou desordens que se situam sob a jurisdição médica, o que causa a desnaturalização da vida cotidiana e o poder emblemático que os profissionais da medicina detêm (Vargens \& Progianti, 2004). Na área obstétrica, esta medicalização ocasionou a representação da gestação e do parto como doença e gerou práticas restritivas ao exercício da autonomia da mulher e de seus direitos reprodutivos e sexuais, bem como o abuso de intervenções desnecessárias (Moura, Lopes, \& Santos, 2009).

Para enfrentar essa problemática, as políticas de saúde visavam à melhoria da qualidade da assistência para atender as necessidades das mulheres, o que demandava mudanças na cultura assistencial e reformas na estrutura física dos serviços de saúde, de modo a oferecer um ambiente institucional afável e adequado; garantir a participação da mulher durante todo o processo assistencial, prestar uma assistência baseada na perspectiva de gênero e com respeito à autonomia feminina e à fisiologia do processo do parto, o que exigiu modificações na formação e atuação dos profissionais de saúde (Narchi, Cruz, \& Gonçalves, 2013).

Dentre as ações do Ministério da Saúde para a qualificação profissional, destaca-se o financiamento dos cursos de especialização em Enfermagem Obstétrica por meio de convênios com as escolas ou faculdades de Enfermagem a partir de 1999, com carga horária total de 400 a 600 h. Para a realização desses cursos, a Área Técnica da Saúde da Mulher constituiu uma Comissão de Especialistas em Enfermagem Obstétrica, em 1999.

Essa comissão adotou os critérios da qualificação profissional propostos pela Associação Brasileira de Obstetrizes e Enfermeiros Obstetras (ABENFO) que estabeleciam a perspectiva de gênero e da humanização da assistência no programa de ensino e os indicadores a serem alcançados pelas alunas durante o curso, como a assistência de no mínimo de 20 partos normais, 15 acompanhamentos de pré-natal, 15 cuidados imediatos aos recém-nascidos, entre outros indicadores (Sacramento, \& Tyrrell, 2006). 
Ressalta-se que outros incentivos ministeriais foram estabelecidos para a melhoria da assistência obstétrica e incentivo da atuação das enfermeiras obstétricas na assistência ao prénatal e ao parto normal, como a Portaria GM n ${ }^{\circ}$ 2815/1998, que incluiu o procedimento parto sem distocia, realizado por enfermeira obstétrica no Sistema de Informações Hospitalares (SIH) do SUS; a Portaria GM n ${ }^{\circ}$ 163/1998, que estabeleceu o laudo de internação de Enfermagem para emissão de Autorização de Internação Hospitalar (AIH) no parto normal, e a Portaria GM n. ${ }^{\circ}$ 985/1999, que instituiu os Centros de Parto Normal (CPN) no sistema de saúde brasileiro, facultando o seu funcionamento integrado ou distanciado fisicamente do hospital de referência e com a equipe profissional mínima composta por enfermeiras obstétricas.

Nesse contexto em transformação e favorável à atuação da Enfermagem Obstétrica no Brasil, a SMS-RJ criou o Programa de Qualificação da Assistência Perinatal na Cidade do Rio de Janeiro, em 2002, que estimulava a assistência ao parto de baixo risco por enfermeiras obstétricas e previa a criação de dez Casas de Parto no município. Inicialmente, houve a liberação de recursos financeiros ministeriais para a construção de três casas de parto. Contudo, houve dificuldades no andamento dessas ações, como a forte resistência das corporações médicas a essas proposições e o escasso quantitativo de enfermeiras estatutárias e especialistas na área obstétrica da rede municipal de saúde. Quando estas profissionais especialistas eram encontradas nas maternidades municipais, grande parte não atuava na assistência direta ao parto normal (Pereira \& Moura, 2009). Essa carência de enfermeiras obstétricas capacitadas para atuar na assistência ao parto normal foi um dos principais motivos para a proposição do curso de especialização na modalidade de residência pela gestão da SMSRJ. Estas profissionais eram consideradas estratégicas para o avanço do seu plano de metas para a área obstétrica e perinatal, como expressa o seguinte depoimento:

[...] Na verdade, tínhamos uma necessidade de ter uma Enfermagem qualificada para a área materno-infantil na rede municipal de saúde. E aí, a partir dessa nossa gestão, começamos a pensar em como qualificar [as enfermeiras] e a conversar sobre isso com a UERJ (...). Percebeu-se que era muito difícil captar enfermeiras obstétricas capacitadas para atuação nesses cenários, casas de parto $e$ mesmo nos centros obstétricos das maternidades do município. (E5, coordenação da SMS)

Além desses fatores logísticos da assistência obstétrica, a SMS-RJ já financiava bolsas para a qualificação de enfermeiras na modalidade de residência, em parceria com Universidade Federal do Estado do Rio de Janeiro (UNIRIO). Portanto, havia uma expertise prévia desta Secretaria na oferta de programas de residência em suas unidades de saúde, como é explicado na seguinte fala:

[...] Aí, o grupo gestor da área da mulher, alguns representantes da gerência da saúde da criança e a superintendência de saúde coletiva se reuniram. E achamos que seria interessante propor uma especialização em enfermagem obstétrica na modalidade de residência, porque o município já tinha esse modelo de formação. Assim, tínhamos a residência médica e também a residência de enfermagem $e$ outras residências. $A$ de enfermagem era em parceria com a UNIRIO. (...) Então, nós tínhamos um diagnóstico de que a Faculdade de Enfermagem da UERJ já dispunha de uma especialização em Enfermagem Obstétrica reconhecida, que existia há muito tempo e com bons resultados. Então, a coordenadora tinha um contato muito grande com a diretora da faculdade e pediu que eu marcasse reunião para pensarmos nas estratégias de implantação da residência. (E3, coordenação da SMS).

A parceria com a unidade acadêmica era fundamental para garantir a certificação das enfermeiras ao término do curso, visto que essa prerrogativa acadêmica e legal é necessária para atuação na especialidade, conforme estabelece a Lei do Exercício Profissional da Enfermagem, $\mathrm{n}^{\circ}$ 7.498/1986.

No âmbito da ENF/UERJ, os docentes percebiam a criação do curso de residência como uma oportunidade de ampliação da atuação das enfermeiras obstétricas na rede pública de saúde e, com isso, um momento favorável para o fortalecimento da Enfermagem nessa área assistencial:

[...] E, por outro lado também, a construção de uma Enfermagem Obstétrica, de um componente da Enfermagem mais apropriado e com mais autonomia para as práticas de atenção ao parto, o cuidado com o 
bebê recém-nascido e, ao mesmo tempo, para que ela [enfermeira] pudesse ter habilidade em lidar com um campo que estava em construção, que era um campo, ainda é, hoje, talvez menos, não sei, um campo bastante inóspito, bem conflituoso. (E.7, docente).

A percepção de que essa modalidade de residência pode conferir melhores competências, habilidades e atitudes necessárias para exercer a especialidade, com mais autonomia e possibilidades de enfrentamento das adversidades da área obstétrica, emergiu como uma motivação subjacente para a criação do curso.

Essa percepção pode estar relacionada ao fato de que as enfermeiras egressas dos cursos de especialização em Enfermagem Obstétrica enfrentam barreiras no exercício da especialidade, como o mercado de trabalho restrito, a resistência médica e o baixo reconhecimento profissional. Essas barreiras se acentuam quando elas atuam na assistência direta ao parto normal, em virtude de que há cursos de curta duração que oferecem um quantitativo insuficiente de experiências práticas e, consequentemente, há aquisição inadequada de habilidades e segurança técnicas necessárias para o atendimento ao parto normal e nascimento (Amorim \& Gualda, 2011).

O contexto acadêmico local também favoreceu a criação da residência, visto que havia ocorrido uma reforma curricular do curso de graduação em Enfermagem no final da década de 1990. O novo currículo era norteado na pedagogia crítica e destacava a relação teoria e prática, dando ênfase para a vivência do aluno na realidade assistencial à saúde desde os períodos iniciais da graduação. Logo, a interação do corpo docente nos serviços de saúde era fundamental para dar vitalidade a essa proposta curricular, e o projeto da residência era mais um componente contribuinte para o fortalecimento do próprio ensino durante o curso de graduação de Enfermagem, como expressa o depoimento da docente:

[...] Havia a questão da integralidade do cuidado e de integrar a prática e a teoria muito fortes [no currículo da graduação] e de estarmos inseridos nos serviços, quer dizer, os professores ficam lá nos serviços com os residentes e com isso também favorecia a presença dos alunos de graduação. Então, permitia uma integração da graduação e da pósgraduação. (E.6, docente).

$\mathrm{Na}$ elaboração do curso de residência surgiram desafios, tais como a formalização de convênio interinstitucional; o trâmite administrativo e legal indispensável para a concretização da proposta de qualificação nas estruturas organizacionais e administrativas da universidade; a constituição do corpo docente e de preceptoras, e a própria inserção das enfermeiras residentes nos serviços obstétricos.

[...] Na época, foi uma proposta nova, inédita na UERJ, não havia nenhuma especialização na modalidade de residência de Enfermagem. Logo, houve muitos desafios durante sua criação, como o reconhecimento acadêmico; se as unidades e os gestores locais iriam receber esse projeto; convencer de sua importância no departamento [da faculdade] para, de fato, terem as respostas do projeto interinstitucional. Havia uma parceria que precisava ser formalizada. E isso foi conseguido internamente, mas não foi muito fácil. Também como se daria o processo de avaliação, como iria ocorrer o acompanhamento e a inserção dessas estudantes.... (E.1, docente).O projeto do curso de residência tinha que ser acolhido pelos professores e ser constituído o corpo docente responsável pela sua execução, com destinação de carga horária dos professores para operacionalizar as disciplinas e a vinculação dos docentes com os serviços de saúde que seriam os campos da prática.

[...] Tínhamos desafios internos na faculdade [de enfermagem] porque era mais um curso que nós abríamos e que exigia uma grande parcela da carga horária docente, um comprometimento maior dos professores $e$ trabalho mais árduo por parte deles. (E.4, docente).

Na instância da SMS também existiram dificuldades de ordem política, administrativa e financeira enfrentadas durante a elaboração da proposta de residência para a criação das vagas entre as já existentes nesta Secretaria e a garantia de recursos para pagamento das bolsas de estudos para as enfermeiras residentes. Dentre as dificuldades, havia aquelas operacionais relacionados à inserção das residentes nas maternidades selecionadas como cenários do ensino em serviço e onde elas seriam inseridas 
na equipe assistencial, como menciona a docente:

[...] Nós tivemos desafios políticos também porque, apesar de nós estarmos conseguindo moldar a política [assistencial], aquela ideia [de estimular a Enfermagem Obstétrica] não era hegemônica. Então, ainda havia uma resistência grande da própria entidade da classe dos médicos. (E.4, docente).

Nestes serviços, as enfermeiras residentes integrariam à equipe de trabalho da instituição. No entanto, era necessário que elas dispusessem de certa autonomia na condução dos cuidados no parto normal. A residência tinha uma proposta de formação que se diferenciava da assistência obstétrica dominante nas maternidades e centrada nas condutas demandas pelo médico.

A superação dos conflitos e desconfianças acerca da atuação das enfermeiras na assistência ao parto normal foi um desafio recorrente durante a criação do curso na modalidade de residência. Portanto, a inserção das enfermeiras residentes nesse contexto adverso dos centros obstétricos das maternidades municipais também fortaleceu a própria luta da Enfermagem Obstétrica para garantir o seu exercício profissional e estabelecer os cuidados preconizados pela política de humanização do parto e nascimento (Progianti, \& Mouta, 2009).

\section{CONSIDERAÇÕES FINAIS}

O estudo sobre o processo de criação do curso de especialização na modalidade de residência em Enfermagem Obstétrica da UERJ/SMS-RJ revelou que as motivações de sua criação estavam relacionadas com as políticas de saúde da época que intentavam modificar o modelo obstétrico medicalizado nos serviços de saúde. Estas políticas incentivaram a inserção de enfermeiras obstétricas na assistência ao parto normal para instituir os cuidados humanizados, estimular o parto normal e melhorar os indicadores da assistência materna e neonatal.

O grupo docente-assistencial que participou do processo de criação do curso de residência almejava qualificar enfermeiras obstétricas para atuar no cuidado ao pré-natal, parto, nascimento e puerpério, com respeito à fisiologia feminina e observância dos princípios da humanização da assistência nas maternidades públicas municipais e nas Casas de Parto, que estavam em fase de implementação no município do Rio de Janeiro.

A inserção das enfermeiras residentes no cotidiano dos serviços foi desafiadora para os docentes e os gestores da rede municipal de saúde pelo fato do modelo assistencial dominante ser medicalizado e em virtude da resistência dos profissionais de saúde, sobretudo os médicos. Além disso, no âmbito da ENF/UERJ também houve dificuldades relacionadas com a constituição do corpo docente responsável pelo desenvolvimento do curso; os trâmites acadêmicos e administrativos para a formalização de convênio interinstitucional com a SMS-RJ, e oficialização do curso de pós-graduação lato sensu na modalidade de residência na universidade, dificuldades estas que foram suplantadas no decorrer da implementação do curso.

\section{REFERÊNCIAS}

Amorim, T., \& Gualda, D. M. R. (2011). Coadjuvantes das mudanças no contexto do ensino e da prática da enfermagem obstétrica. Rev Rene, 12(4), 833-840.

SECRETARIA DE GESTÃO DO TRABALHO E DA EDUCAÇÃO NA SAÚDE (2012). Edital $\mathrm{n}^{0}$ 21, de 5 de setembro de 2012 (BR). Processo seletivo destinado à oferta de bolsas para 0 Programa Nacional de Residência em Enfermagem Obstétrica (PRONAENF). Diário Oficial da União. Recuperado de https://www.jusbrasil.com.br/diarios/40314104/d ou-secao-3-06-09-2012-pg-136.

Lei $n^{0}$ 11.129, de 30 de junho de 2005(2005). Institui o Programa Nacional de Inclusão de Jovens - ProJovem, cria o Conselho Nacional da Juventude - CNJ e a Secretaria Nacional de Juventude, altera as Leis no s 10.683 e 10.429 e dá outras providências. Brasília, DF: CNJ Recuperado

de http://www.planalto.gov.br/ccivil_03/_Ato20042006/2005/Lei/L11129.htm

Ministério da Saúde. Programa de humanização no pré-natal e nascimento, 2000. Brasília: Ministério da Saúde.

Costa, A. A. N. M., \& Schirmer, J. (2012). A atuação dos enfermeiros egressos do curso de especialização em obstetrícia no nordeste do Brasil: da proposta à operacionalização. EsC Anna Nery Rev Enferm, 16(2), 332-339.

Erdmann, A. L., Fernandes, J. D., \& Teixeira, G. A. (2011). Panorama da educação em enfermagem no Brasil: graduação e pós- 
graduação. Enfermagem em Foco, 2(Supl. 1),8993.

Lima, G. P. V., Pereira, A. L. F., Guida, N. F. B., Progianti, J. M., Araújo, C. L. F., \& Moura, M. A. V. (2015). Expectativas, motivações e percepções das enfermeiras sobre a especialização em enfermagem obstétrica na modalidade residência. Esc Anna Nery Rev Enferm,19(4), 593-599.

Minayo, M. C. S. (2006). O desafio do conhecimento: pesquisa qualitativa em saúde. São Paulo: Hucitec.

Moura, C. F. S., Lopes, G. T., \& Santos, T. C. F. (2009). Humanização e desmedicalização da assistência à mulher: do ensino à prática. Rev enferm UERJ, 17,(2),182-187.

Narchi, N. Z., Cruz, E. F., \& Gonçalves, R. (2013). O papel das obstetrizes e enfermeiras obstetras na promoção da maternidade segura no Brasil. Ciênc saúde coletiva, 18(4),1059-1068.

Oliveira, A. S., Damasceno, A. K. C., Moraes, J. L., Moreira, K. A. P., Teles, L. M. R., \& Gomes L. F. S. (2014). Technology used by companions in labor and childbirth: a descriptive study. Online braz j nurs,13(1), 36-45. Recuperado de http://www.objnursing.uff.br/index.php/nursing/ article/view/4254

Pereira, A. L. F., \& Nicácio, M. C. (2014). Formação e inserção profissional das egressas do curso de residência em enfermagem obstétrica. Rev enferm UERJ, 22(1), 50-60.

Pereira, A. L. F. (2013). As Políticas Públicas brasileiras para a formação e qualificação das enfermeiras para a assistência ao parto e nascimento: a experiência de formação, inserção e fixação de enfermeiras obstétricas no município do Rio de Janeiro. Anais do VIII COBEON, Florianópolis, SC, Brasil.

Pereira, A. L. F., \& Moura, M. A. V. (2009). Hegemonia e contra hegemonia no processo de implantação da Casa de Parto no Rio de Janeiro. Rev Esc Enferm USP,43(4), 872-879.

Progianti, J. M., \& Mouta, R. J. O. (2009). A enfermeira obstétrica: agente estratégico na Implantação de práticas do modelo humanizado em Maternidades. Rev enferm UERJ, 17(2), 165169.

Rattner, D. (2014). Da Saúde Materno-Infantil ao PAISM. Tempus, actas de saúde colet, 8 (2), 103-108.

Rosa, S. D., \& Lopes, R. E. (2009). Residência Multiprofissional em Saúde e Pós-Graduação Lato Sensu no Brasil: apontamentos históricos. Revista Trabalho, Educação, Saúde, 5(3),479498.

Sacramento, M. T. P., \& Tyrrell, M. A. R. (2006). Vivências das enfermeiras nos cursos de especialização em enfermagem obstétrica. Rev Enferm UERJ, 14(3), 425-433.

Silva, M. G., Fernandes, J. D., Teixeira, G. A. S., \& Silva, R. M. O. (2010). Processo de formação da(o) enfermeira(o) na contemporaneidade: desafios e perspectivas. Texto Contexto-Enferm, 19(1), 176-184.

Souza, M. H. N., \& Tyrrell, M. A. R. (2011). Políticas de salud a la mujer en Brasil, 19742004 / Políticas de saúde para a mulher no Brasil, 1974-2004. Rev enferm UERJ, 19(1), 7076.

Vargens, O. M. C., \& Progianti, J. M. (2004). O processo de desmedicalização da assistência à mulher no ensino de enfermagem Rev Esc Enferm USP, 38(1),46-50.

\section{AGRADECIMENTO}

Ao Conselho Nacional de Desenvolvimento Científico e Tecnológico-CNPq pelo apoio financeiro à pesquisa. 